CEIR-TH-5811/90

\title{
SIGNAIS FOR HEAVY EXOTICS AT HADRON COLLIDERS AND SUPERCOLLIDERS
}

\author{
M. Drees \\ CERN - Geneva \\ and \\ X. Tata \\ Department of Plysies and Astronomy, \\ University of Hawaii at Manoa, Honolulu, USA
}

\begin{abstract}
ABSTRAC'T
We sturly the signals for the production of heavy long-lived charged particles at present and future hadron colliders. The most important signature for these is their large penetration and velecities $\beta$ significantly smaller than 1 , but we also comment on purely calorimetric signatures. We point ont that the CDF Collaboration slomlel already be able to derive significant coustraints on the procluction of scalar or fermionic long-lived colour-triplet particles, from its presenti data sample, which has an integrated luminosity of $5 \mathrm{ph}^{-1}$.
\end{abstract}

CERN-TH-5811/90

July 1.990 


\title{
Signals for Heavy Exotics at Hadron Colliders and Supercolliders
}

\author{
M. Drees \\ CERN, Geneva, Switzerland \\ X. Tata \\ Department of Physics and Astronomy, \\ University of Hawaii at Manoa, Honolulu, USA
}

\begin{abstract}
We study the signals for the production of heavy long-lived charged particles at present and future hadron colliders. The most important signature for these is their large penetration and velocities $\beta$ significantly smaller than 1 , but we also comment on purely calorimetric signatures. We point out that the CDF Collaboration should already be able to derive significant constraints on the production of scalar or fermionic long-lived colour-triplet particles, from its present data sample, which has an integrated luminosity of $5 \mathrm{pb}^{-1}$.
\end{abstract}

Although experimentally the Standard Model (SM) looks better than ever [1], there are good theoretical reasons [2] for believing that it is not the whole story. Almost all extensions of the SM predict the existence of new, charged, and/or coloured particles. These particles, if they exist, have to be quite heavy to have escaped detection up to now. In general, such heavy, strongly interacting or charged particles decay almost instantaneously after production, so they do not leave a track within the detector. Extensive searches for such unstable particles have been performed at $\mathrm{e}^{+} \mathrm{e}^{-}$colliders [3] (SLC, LEP) as well as at $\mathrm{p} \overline{\mathrm{p}}$ colliders [4] (SPS, Tevatron), but with a null result. Under certain circumstances, however, these particles can be long-lived or even stable; in the latter case, their decay is usually forbidden by some symmetry. Ilowever, a fairly large number of any particles with a lifetime bigger than $O\left(10^{9}\right.$ years $)$ should have survived from their production during the Big Bang into our present epoch, where they would have formed exotic nuclear isotopes; experimental upper bounds on the abundance of such isotopes therefore essentially rule out [5] models with very long-lived charged and/or coloured particles with masses in the range between a few tens of $\mathrm{GeV}$ and a few tens of $\mathrm{TeV}$. For collider expcriments, however, a particle is 'stable' if it lives longer than $O\left(10^{-7}\right)$ seconds, which is more than 
20 orders of magnitude shorter than the minimal lifetime of a cosmologically stable particle [6].

Two classes of models can predict long-lived massive charged particles: models with a weakly broken symmetry, where the particle would be stable if the symmetry were exact; and models with an exact symmetry which forbids the decay of heavy exotics into ordinary particles, where the decay of the charged particle into yet another neutral particle (allowed by the symmetry) is suppressed either by small couplings or by phase space. Examples of models of the first kind are supersymmetric models [7] with weakly broken R-parity. Examples of the second kind are supersymmetric models with exact R-parity, where the lightest supersymmetric particle is the gravitino [8] (the superpartner of the graviton) or some other 'hidden sector' particle that couples only gravitationally to the superpartners of 'ordinary' particles. In this case the lifetime of a scalar exotic $\tilde{\mathrm{f}}$, which decays dominantly into a longitudinal gravitino via $\tilde{\mathrm{f}} \rightarrow \mathrm{f} \tilde{\mathrm{G}}$, is of the order of $10^{4} \mathrm{~s}\left(100 \mathrm{GeV} / m_{\mathrm{f}}\right)^{5}\left(m_{\mathrm{G}} / 1 \mathrm{GeV}\right)^{2}$, which is clearly within the-for us-interesting range. Another example could be the recently proposed [9] 'pscudo-IIiggs' model, with an absolutely stable neutral pscudo-lliggs and a possibly very small mass gap to the lightcst charged one.

In order to be as model-independent as possible, we consider four different types of particles: colour-singlet charge- 1 scalars $\left(\tilde{e}_{R}\right)$ and fcrmions $\left(\tilde{W}_{-}\right)$, as well as colour-triplet scalars $(\tilde{t})$ and fermions (D). The notation for the first three particles is inspired by supersymmetry, $\tilde{W}_{-}$being the lightest chargino, $\tilde{e}_{R}$ an $S U(2)$ singlet cliarged slepton, and $\tilde{\mathrm{t}}$ a scalar top; D could be any exotic quark, as present, for example, in $\mathrm{E}(6)$ models [10]. (Although a fermion, D could still have odd R-parity, and hence be long-lived as discussed above.) Even though our choice of exotics is motivated by candidates for the lightest charged particle in supersymmetric models, it should be recognized that this is only for illustration, and that our considerations are quite general.

The detection of any of these particles at an $\mathrm{e}^{+} \mathrm{e}^{-}$collider is straightforward, since they all have full gauge-strength couplings to the photon and the $\mathrm{Z}$ boson; the signal would be a highly penetrating particle (see below) whose velocity $\beta$ is less than 1 and whose momentum $p$ is less than the beam encrgy. So far, only the Mark II Collaboration has published [11] bounds on such particles from searches on the $\mathrm{Z}$ pole, but the fact that none of the LEP groups, with their much larger data samples, has announced a signal can be interpreted, we believe, as a lower bound of about $m_{Z} / 2$ on the mass of any of these candidates. For the remainder of this letter we will therefore focus on the signals of long-lived charged particles at hadron colliders.

The CDF Collaboration has already published [12] a bound on the crosssection for long-lived charged particles. IIowever, since this search was based only on their 1987 data sample, corresponding to an integrated luminosity of $26.2 \mathrm{nb}^{-1}$, this bound does riot constrain any of the four models we are considering. [It does constrain models with long-lived particles in higher representations of SU(3).] Nevertheless Ref. [12] is very valuable for us, since it describes the 
cuts applied to extract the signal.

Again, the basic idea is to look for a penetrating particle with a velocity $\beta$ that is significantly less than 1 . These particles are penetrating essentially for the same reason that muons are penetrating. The energy loss $\mathrm{d} E / \mathrm{d} x$ of a charged particle through ionization of the matter it traverses only depends [13] on the velocity $\beta$, not on the mass $m$, of the particle. Since the kinetic energy is proportional to $m$, this means that the fractional energy loss or $\mathrm{d} \beta / \mathrm{d} x$ is proportional to $1 / m$. As a result, the penetration length $L$ of a charged particle in a given material is proportional to $m$.

In Fig. 1 we show the energy loss $\mathrm{d} E / \mathrm{d} x$ through ionization in iron, as given by the well-known Bethe-Bloch formula, as well as the particle's velocity $\beta$, as a function of the distance $x$ to the point where the particle is stopped. The penetration length of a particle with initial velocity $\beta$ can also be read off this plot. Typical detectors will have roughly one or a few metres of iron-equivalent between the interaction point and the muon counters, which will be used for the trigger. From Fig. 1 we see that a particle of a mass of the order of $100 \mathrm{GeV}$ will need an initial $\beta$ of roughly 0.5 in order to penetrate a few metres of iron. For more precise estimates, the following expression can be used for the penetration length in iron:

$$
L / M=0.039 \mathrm{~cm} / \mathrm{GeV} \cdot \mathrm{e}^{8.47 \beta} ;
$$

this reproduces the exact numerical result to $\sim 10 \%$ for $\beta$ between 0.4 and 0.7 .

So far we have only considered the energy loss through ionization. Particles in non-singlet representations of $\mathrm{SU}(3)_{c}$ can also lose energy in hadronic reactions. Of course, such particles will hadronize in $O\left(10^{-23}\right)$ seconds, to form an exotic hadron. Heavy colour-triplet particles will dominantly form exotic 'mesons' by pulling a $\bar{u}$ or a $\bar{d}$ quark out of the vacuum. (Notice that in the case of the scalar quark $\tilde{t}$, the 'meson' will have spin 1/2.) These 'mesons' can lose energy by scattering off the nuclei of the surrounding medium. The energy loss of the exotic meson $\mathrm{F}$ in one such collision is given by

$$
\Delta E=\frac{m_{\mathrm{X}}^{2}-m_{\mathrm{T}}^{2}-t}{2 m_{\mathrm{T}}}
$$

where $t$ is the Mandelstam variable (the squared four-momentum transferred to F), $m_{\mathrm{T}}$ is the mass of the relevant target, and $m_{\mathrm{X}}$ is the mass of the hadronic system (other than the outgoing exotic meson) produced in the collision. For small $|t|$, below $0.01 \mathrm{GeV}^{2}$ or so (corresponding to distances larger than a few fermis), the nuclear structure is not resolved, so $m_{\mathrm{T}}$ is the mass of the nucleus. For larger $|t|$, the scattering involves a discrete nucleon inside the nucleus, so $m_{\mathrm{T}}=m_{\mathrm{N}}$. For elastic scattering, $m_{\mathrm{X}}=m_{\mathrm{T}}$, whereas inelastic scattering is characterized by $m_{\mathrm{X}}>m_{\mathrm{T}}$.

For very large momentum transfer, $|t|>t_{0} \simeq 1 \mathrm{GeV}^{2}$, the relevant process is the scattering of the exotic (s)quark of a parton inside the nucleus. It is easy 
to see that this implies for the Bjorken- $x$ of the probed parton:

$$
x>\frac{\sqrt{t_{0}}}{2 m_{\mathrm{N}} \gamma},
$$

where $\gamma=1 / \sqrt{1-\beta^{2}}$. As a result, this hard contribution to the total inelastic cross-section remains small $(<0.1 \mathrm{mb})$ unless $\beta$ is very close to unity $(\beta \gtrsim 0.98)$. On the other hand, the total elastic cross-section will be dominated by the region of very small $|t|$, since it should fall like $1 / t^{2}$ in the region $|t| \lesssim 0.1 \mathrm{GeV}^{2}$. Therefore, elastic processes will contribute very little to the energy loss of $F$.

This leaves us with soft inelastic scattering. As discussed earlier, the nature of the process depends on the size of $|t|$ : for a momentum transfer that is small compared with the inverse nuclear radius, the exotic meson will scatter off the whole nucleus, while for somewhat larger $|t|$ it will 'see' the nucleons inside the nucleus. From Eq. (2) we see that the energy loss per collision increases with $m_{\mathrm{X}}$ and $|t|$. For given $t, m_{\mathrm{X}}$ is bounded from above by

$$
m_{\mathrm{X}}^{2} \leq m_{\mathrm{T}}^{2}+2 \sqrt{|t|} \sqrt{\gamma^{2}-1} m_{\mathrm{T}}-|t|\left(1+\frac{\gamma m_{\mathrm{T}}}{m}\right),
$$

where terms of order $t / m^{2}$ have been neglected, and $|t|<m_{\mathrm{T}}^{2}\left(\gamma^{2}-1\right)$ has been assumed. If the whole nucleus $\mathrm{A}$ is the target, this condition is always fulfilled; furthermore, in this case the last term is negligible, and one has for the energy loss of $\mathbf{F}$ :

$$
\delta E_{\mathrm{FA}} \leq \sqrt{|t|} \sqrt{\gamma^{2}-1} \frac{1}{R_{\mathrm{A}}} \sqrt{\gamma^{2}-1}
$$

or at most a few tens of $\mathrm{MeV}$ for $\beta \leq 0.8$ or so.

In the case of F-nucleon scattering, Eq. (4) is applicable only for $|t|<$ $m_{\mathrm{N}}^{2}\left(\gamma^{2}-1\right)$; otherwise, $m_{\mathrm{X}}$ is simply bounded by $\sqrt{s}-m_{\mathrm{N}}$, where $\sqrt{s}$ is the centre-of-mass energy. Also, one now has to require that $m_{\mathrm{X}}>m_{\mathrm{N}}+m_{\pi}$, since single-pion production is the lowest possible excitation in this case; this implies $\beta \gtrsim 0.5$. If we simply assume the cross-section to be independent of $m_{\mathrm{X}}$ and $t$ for $|t|<t_{0}$ and to vanish for $|t|>t_{0}$, we find that the average energy loss per collision can be parametrized by

$$
\left\langle\delta E_{\mathrm{FN}}\right\rangle=\frac{\sqrt{t_{0}}}{2} \frac{\gamma^{2}}{\gamma+\frac{1}{3}} .
$$

This gives $\langle\delta E\rangle \simeq 0.5 \mathrm{GeV}$ for $\beta=0.7$, and $\langle\delta E\rangle \simeq 1 \mathrm{GeV}$ for $\beta=0.9$, even if $\sqrt{t_{0}}$ is as large as $1 \mathrm{GeV}$.

In order to estimate the hadronic energy loss in material we also have to estimate the relevant interaction length. For ultrarelativistic protons in iron, the nuclear interaction length [14] is $17 \mathrm{~cm}$. Since the exotic meson contains only one light quark, from the additive quark model one would expect its interaction length to be three times larger. We then see that even if all these reactions 
are $\mathrm{F}$-nucleon reactions, hadronic energy losses cannot play a significant role in reducing the total absorption length for $\beta \lesssim 0.6$. Since a particle with $\beta=0.6$ and mass $m>50 \mathrm{GeV}$ can penetrate more than $3 \mathrm{~m}$ of iron (see Fig. 1), hadronic energy losses will not significantly reduce the signal of penetrating particles. We have, nevertheless, discussed them in some detail here since they could contribute to the calorimetric signatures of such particles.

Before discussing such signatures, we return to the computation of the rate of penetrating events of the type the CDF Collaboration has searched for [12]. Four different cuts have to be applied to isolate this signal:

$$
\begin{aligned}
\beta & <\beta_{\max }, \\
p_{\mathrm{T}} & >p_{\text {Tmin }}, \\
|\cos \theta| & <|\cos \theta|_{\max }, \\
L_{\text {abs }} & >d_{\mathrm{Fe}} .
\end{aligned}
$$

The first cut guarantees that the exotic, heavy particle reaches the outer detector components, where the time of flight is measured, significantly later than do ultrarelativistic particles. The second cut helps to suppress 'soft' muons. The third cut is simply due to the finite angular acceptance of the muon counters. Finally, the fourth cut guarantees that the particle can penetrate the muon chambers; for this cut, we use the approximation (1) for the absorption length in iron.

The computation of the cross-section after cuts is now fairly straightforward. For the production of the $\mathrm{SU}(2)$ singlet scalar $\tilde{e}_{\mathrm{R}}$ we include $\gamma$ and $\mathrm{Z}$ exchange graphs [15]. Since the $\tilde{W}_{-}$is in general a mixture of states in different SU(2) representations [7], conservatively we only include $\gamma$-exchange in this case. For the production of scalar and fermionic quarks we include the usual leading-order $\mathrm{q} \tilde{\mathrm{q}}$-annihilation and gg-fusion processes; the relevant cross-sections can be found in Refs. [16] and [17] for quarks and squarks, respectively.

For these last two particles, the cross-section has to be corrected for hadronization effects. Since the fragmentation functions for heavy quarks are expected [18] to be very hard, the difference between the velocity of the produced (s)quark and the velocity of the exotic meson should be very small. However, some of the exotic (s)quarks will hadronize into neutral mesons, which do not contribute to the signal since they do not produce a track in the inner part of the detector. If only $u$ and $d$ (anti)quarks are relevant in this hadronization process, this effect will reduce the event rates for our hadronic exotics by a factor of 2; this factor will be somewhat different if a significant fraction of the exotic (s)quarks hadronize into strange mesons, which will be charged (neutral) for exotics with charge $\frac{2}{3}\left(\frac{1}{3}\right)$. Furthermore, inelastic hadronic reactions can change the charge of the exotic meson; this effect reduces the signal by another factor of between 1 and 2. [Note that strong interactions cannot destroy a long-lived heavy (s)quark.] We have not included these factors in the cross-sections shown below; therefore, these have to be divided by a factor of between 1.5 and 4 , de- 
pending on the hadronization and the thickness of matter in front of the muon chambers.

In Figs. 2a-c we present total cross-sections for the production of the four exotic particles we are considering for various combinations of accelerators and detectors, where the latter are defined by the four cuts of Eqs. (7). We restrict ourselves to the mass range $m<300 \mathrm{GeV}$ since heavier long-lived particles with unit charge appear very unnatural to us. After all, most extensions of the SM are constructed with a view to explaining electroweak gauge-symmetry breaking, or at least making it technically natural [19]; it seems, therefore, very unlikely that the lightest new particle that has gauge interactions, which, as explained earlier, is the only one that can be expected to be long-lived, should be heavier than a few hundred $\mathrm{GeV}$.

In Fig. 2a we used the values of $\beta_{\max }, p_{\text {rmin }}$, and $|\cos \theta|_{\max }$ specified by the CDF Collaboration [12]. The iron thickness of $84 \mathrm{~cm}$ corresponds to 5 nuclear interaction lengths, which is the value quoted in their paper [20]. The lower and upper curves correspond to the present Tevatron collider and a possible future upgrade, respectively. We see that the $1988 / 89$ data sample of $\sim 5 \mathrm{pb}^{-1}$ should be sufficient to search for scalar and fermionic quarks below 125 and $180 \mathrm{GeV}$, respectively, assuming 5 events to be necessary for a signal and a factor of 2 reduction due to the hadronization effects mentioned earlier. If the upgraded Tevatron succeeds in collecting a data sample of several hundred $\mathrm{pb}^{-1}$, even the present CDF detector should be able to cover almost the whole mass range we consider reasonable for the strongly interacting particles. Hlowever, the present data sample docs not allow the bound on long-lived $\tilde{e}_{R}$ and $\tilde{W}_{-}$to be improved beyond the range covered by the SLC and LEP; and even the most optimistically upgraded Tevatron will not be able to extend this range beyond $\sim 80 \mathrm{GeV}$ for $\tilde{e}_{R}$ and $170 \mathrm{GeV}$ for $\tilde{\mathrm{W}}_{-}$. This is partly due to the fact that for $m \lesssim 100 \mathrm{GeV}$ the efliciency is fairly low, since most particles are produced with $\beta>0.7$; the ratio of fast particles increases with $\sqrt{s}$, so that the increase of the total crosssection is partly cancelled by the reduction of the efliciency. This problem is particularly severe for $\tilde{e}_{R}$, which are produced in a p-wave; for $m=50 \mathrm{GeV}$ and $\sqrt{s}=3 \mathrm{TeV}$, only $11 \%$ of all $\tilde{e}_{\mathrm{R}}$ pairs lead to even one particle that passes all cuts.

In Fig. 2b we show results for a 'generic' detector at the proposed supercolliders LHC and SSC. We feel that our choice of cut parameters, and in particular of $\beta_{\max }$, is fairly conservative. Even for this choice of parameters the LIIC should be able to probe the whole mass range even for the $\tilde{e}_{R}$ already alter one year of running at a luminosity of 'only' $10^{33} \mathrm{~cm}^{-2} \cdot \mathrm{s}^{-1}$; the SSC could do the same in about half the time. In fact, this type of search might be difficult to perform at much higher luminosities, where the time between consecutive interactions (i.e. the bunch spacing) becomes smaller than the time a particle needs to leave the detector; this would obviously complicate the interpretation of time-of-flight measurements [21].

At very high luminosities a special-purpose detector might therefore be more 
appropriate. Such a detector has been suggested by Litke et al. [22]. It has a very small angular acceptance, but should be able to detect particles with $\beta$ as large as 0.95 . In Fig. 2c we show cross-sections for penetrating events (upper curves) in this case. We see that the event rate in such a detector is roughly 30 times lower than the one in our 'generic' supercollider detector. At the LHC, such a special detector would therefore have to operate at the full luminosity of $\sim 10^{34} \mathrm{~cm}^{-2} \cdot \mathrm{s}^{-1}$ in order to cover the full mass range for $\tilde{e}_{R}$. Since the SSC cross-section for $\tilde{e}_{\mathbf{R}}$ is again only a factor of 2 above the LIIC cross-section, this detector would need about 5 years at the design luminosity to cover the whole mass range.

Supposing one finds a signal, how could one discriminate between the various candidates? One answer is already obvious from Fig. 2: the cross-sections are very different for the four cases if the mass is kept constant; this mass can be extracted from the measurement of the particle's momentum and velocity.

Another possibility is to study the angular and momentum distributions of the signal events. In Figs. $3 a$ and $b$ we present these distributions for $m=50 \mathrm{GeV}$ and $\sqrt{s}=1.8 \mathrm{TeV}$, where we have used the cuts characteristic for the CDF detector; however, we have not applied the cut on the quantity plotted. (Note that $p / m=\beta \gamma$, so that a cut on $\beta$ directly transiates into a cut on $p / m$.) Nevertheless the combination of the other three constraints imposes some bounds:

$$
\begin{gathered}
\sin ^{2} 0>\frac{p_{\text {Tmin }}^{2}}{m^{2}}\left(\frac{1}{\beta_{\max }^{2}}-1\right) ; \\
p / m>\max \left[\frac{p_{\mathrm{T} \min }}{m}, \frac{1}{\sqrt{1 / \beta_{\mathrm{c}}^{2}}-1}\right],
\end{gathered}
$$

where $L_{\mathrm{abs}}\left(\beta_{\mathrm{c}}\right)=d_{\mathrm{Fe}}$. Unfortunately, we find that the differences between the four cases considered are not very pronounced, although it should still be possible to discriminate bctween the $\tilde{e}_{R}$ and the other three candidates. The reason is that $\tilde{e}_{R}$ has to be produced in a p-wave, i.e. $\mathrm{d} \sigma / \mathrm{d} t \sim p^{3} \sin ^{2} \theta^{*}$, where the asterisk refers to the c.m.s. frame of the $\tilde{e}_{R}$ pair; as a result, large angles and large momenta are dynamically favoured for $\tilde{\mathrm{e}}_{\mathrm{R}}$. The momentum distribution of $\tilde{W}_{-}$is somewhat harder than that of $D$ and $\tilde{t}$, because $\tilde{W}_{-}$can only be produced in $q \tilde{q}$ annihilation; at a $\mathrm{p} \overline{\mathrm{p}}$ collider, the corresponding flux is harder than the $\mathrm{gg}$ flux, which dominates the production of $\mathrm{D}$ and $\tilde{\mathrm{t}}$ pairs. However, this difference is much smaller at the proposed pp supercolliders.

In general the momentum distribution will be softer at larger values of $m / \sqrt{s}$. This increases the differences between the four cases, since it means that a larger part of the curve can be studied for given $\beta_{\max }$. Also, the Lorentz boost from the parton-parton c.m. frame to the laboratory frame, which tends to smear out differences in the angular distributions, tends to decrease for larger $m / \sqrt{s}$, again leading to more distinct distributions. Finally, going to $p p$ colliders tends 
to increase this boost for processes involving $\mathrm{q} \overline{\mathrm{q}}$ annihilation, since this makes the initial state less symmetric, as the $\bar{q}$ now has to be a sea-quark.

We note here that if the energies and momenta of both heavy particles are measured, it is straightforward to construct the angular distribution in the c.m. frame. In this frame, the difference between $\tilde{e}_{\mathrm{R}}$ and the other three cases is somewhat more pronounced. However, the effects of the limits and the integration over a range of c.m. energies again leads to fairly similar distributions for $\tilde{\mathrm{W}}_{-}, \tilde{\mathrm{t}}_{\text {, and }} \mathrm{D}$.

Finally, one might be able to distinguish between hadronic and non-hadronic particles by studying the energy deposition of the candidates in the detector. This would be easier for faster particles, since they can deposit more energy in a single hadronic interaction. The special-purpose detector of Ref. [22] is most suitable (among the three detectors we studied) for this kind of analysis, because of its large $\beta$ acceptance. Heavy exotic mesons with $\beta=0.9(0.95)$ could deposit as much as $3.0 \mathrm{GeV}(6 \mathrm{GeV})$ in a single soft inelastic reaction, as can be seen from Eqs. (2) and (4) assuming $|t|<1 \mathrm{GeV}^{2}$ for this type of reaction. The $3.2 \mathrm{~m}$ of iron calorimeter correspond to about 6 interaction lengths in the additive quark model. This should guarantee scveral interactions with energy loss $>1 \mathrm{GeV}$ for $\beta>0.9$. We recall that the track might actually disappear between two of these interactions, if an initially charged exotic meson was transformed into a neutral one by the first interaction and transformed back into a charged meson by the second scattering. Of course, this kind of study can only be undertaken in a calorimeter with fairly fine longitudinal segmentation. We mention, in passing, that such a calorimeter might cven allow the search for neutral, heavy, strongly interacting particles.

The large iron depth of this special-purpose detector might also make it possible to study an entirely different signature, i.e. to look for heavy particles that get stuck inside the calorimeter. The lower curves in Fig. $2 \mathrm{c}$ show the cross-sections for the case where at least one particle does not penetrate the calorimeter all the way to the muon chambers. We see that the cross-section is, in principle, sufficiently large, except for very light particles and for $\tilde{e}_{R}$. The inefficiency in detecting light particles in this way is due to the rather stiff $p_{\mathrm{T}}$ cut, which forces light particles to be fast and hence penetrating; this problem could be solved by relaxing that cut somewhat. Ilowever, this would still not allow $\tilde{e}_{R}$ to be detected in this fashion, owing to the tendency of these particles to be produced with large momenta, as discussed above.

From Fig. 1, we see that a particle that is stopped in the calorimeter deposits at least $\sim 0.4 \mathrm{GeV}$ in the last $\mathrm{cm}$ of its track; heavier particles deposit more. The energy deposition per unit length is at least 10 times that of a minimum ionizing track for $0.1 \mathrm{~cm} \cdot m$ ( $m$ in GeV), or about $10 \mathrm{~cm}$ for a particle of mass $100 \mathrm{GeV}$. Again a fine longitudinal segmentation of the calorimeter is necessary to look for this signal.

To summarize: We have studied the production of long-lived heavy charged particles at prescent and future hadron colliders. We found that the CDF Col- 
laboration should be able to put significant bounds on such particles by looking for penetrating, slow particles in its present data sample, if they have strong interactions. For particles without strong interactions one needs $\mathrm{e}^{+} \mathrm{e}^{-}$or pp supercolliders to cover the whole range of masses that might be considered reasonable in extensions of the Standard Model which aim to shed some light on the mechanism of electroweak symmetry breaking. We find that this can be successfully done within one year by a multipurpose detector already at a relatively low luminosity $\left(10^{33} \mathrm{~cm}^{-2} \cdot \mathrm{s}^{-1}\right.$ for the LHC, roughly half as much for the SSC.) Alternatively, one could use a specialpurpose detector at full LHC luminosity $\left(\gtrsim 10^{34} \mathrm{~cm}^{-2} \cdot \mathrm{s}^{-1}\right)$. In addition to being much less expensive, this latter detector would have the advantage that it might allow the study of other, calorimetric signatures of such particles.

\section{References}

[1] For a recent review, see for example G. Altarelli, Invited talk given at the Neutrino 90 Conference, CERN, Geneva, 1990.

[2] For a review, see J. Ellis, preprint CERN TH.3718 (1983).

[3] For a recent review, see M. Oreglia, Invited talk given at the Neutrino 90 Conference, CERN, Geneva, 1990.

[4] See, for example, the talks by J.M. Gaillard and L. Nadulman given at the EPS Conference on High-Energy Physics, Madrid, 1989.

[5] P.F. Smith and J.R.J. Bennet, Nucl. Phys. B149, 525 (1979); P.F. Smith et al., Nucl. Phys. B206, 333 (1982);

E. Norman, S.B. Gazes and D.A. Bennett, Phys. Rev. Lett. 58, 1403 (1987).

[6] Cosmology will most likely also constrain some particles with shorter lifetimes, since their decay could distort the $3 \mathrm{~K}$ background radiation and/or primordial synthesis. They can also be produced in very energetic cosmicray interactions, increasing their present abundance on the Earth beyond that of Big. Bang relics. However it seems plausible to assume that for most models, some lifetimes $>10^{-5} \mathrm{~s}$ will not be excluded by these arguments.

[7] For a review, see II.P. Nilles, Phys. Rep. 110, 1 (1984).

[8] A superlight gravitino with a mass of $1 \mathrm{eV}$ or less was suggested by J. Ellis, K. Enqvist and D.V. Nanopoulos, Phys. Lett. B147, 99 (1984) and B151, 357 (1985). For our purpose its mass would have to be at least of $O(1 \mathrm{keV})$; a much heavier gravitino would serve as well, as long as it is lighter than the lightest sparticle of the visible sector.

[9] K. Griest and M. Sher, Phys. Rev. Lett. 64, 135 (1990). 
[10] For a review, see J. Ilewett and T. Rizzo, Phys. Rep. 183, 193 (1989).

[11] E. Soderstrom et al., Stanford preprint SLAC-PUB-5192 (1990).

[12] F. Abe et al., Phys. Rev. Lett. 63, 1447 (1989).

[13] U. Fano, Annu. Rev. Nucl. Sci. 13, 1 (1963)

[14] Particle Data Group, Phys. Lett. B204, 1 (1988).

[15] It has recently been claimed by S.K. Kim et al., Z. Phys. C46, 267 (1990), that the total cross-section for the pair production of charged scalars at pp supercolliders is dominated by $\gamma \gamma$ fusion. Ilowever, we found the $\gamma \gamma$ cross-section to be at least one order of magnitude below the annihilation cross-section, and have therefore ignored this contribution. We thank J.A.M. Vermaseren for an independent check of our calculation.

[16] B.L. Lombridge, Nucl. Phys. B151, 429 (1979).

[17] P.R. IIarrison and C.II. Llewellyn Smith, Nucl. Phys. B213, 223 (1983); erratum, Nucl. Pliys. B223, 542 (1983).

[18] D.A. Morris, Nucl. Phys. B313, 634 (1989), and references therein.

[19] G. 't Ilooft, in Recent, Developments in Gauge Theories, Proc. Cargèse Summer Institute, 1979, eds. by G. 't Ilooft et al., NATO Advanced Study Institute, Series B, Vol. 59 (Plenum, New York, 1980), p. 101.

[20] The effects of absorption of the exotic particles inside the calorimeter were apparently not included in the analysis of Ref. [12]. They do include a cut on the minimal allowed $\beta$, but quote a value of 0.2 for this cut for heavy particles; however, Fig. 1 shows that even a $300 \mathrm{GeV}$ particle with $\beta=0.2$ is stopped in less than $30 \mathrm{~cm}$ of iron. We estimate that inclusion of this absorption effect decreases the cross-section by $\sim 10 \%$ for particles with mass around $300 \mathrm{GeV}$.

[21] S. Errede et al., Proc. DPF Summer Study on the Design and Utilization of the Superconducting Super Collider, Snowmass, Colo., 1984.

[22] A.M. Litke et al., Proc. Workshop on Experiments, Detectors and Experimental Areas for the Supercollider, Berkeley, 1987, eds. R. Donaldson and M.G.D. Gilchriesc (World Scientific, Singapore, 1988), p. 853. 
Fig. 1: The energy loss through ionization $\mathrm{d} E / \mathrm{d} x$ and the velocity $\beta$ of a particle with mass $m$ and unit charge as a function of the rescaled distance $x / m$ from the point where the particle will be stopped in iron; notice that $x / m$ is independent of $m$.

Fig. 2: Total signal cross-section after cuts at (a) $p \bar{p}$ and (b, c) pp colliders, as a function of the mass $m$ of the long-lived particle. The different textures correspond to different types of particles: $\tilde{e}_{\mathbf{R}}$ (solid) is a spin-0 $\mathrm{SU}(2)$ and colour singlet, $\tilde{W}_{-}$a spin- $-\frac{1}{2}$ colour singlet, $\tilde{\mathrm{t}}$ a spin- 0 colour triplet, and $\mathrm{D}$ a spin- $-\frac{1}{2}$ colour triplet. Note that only one particle of the produced pair needs to pass the cuts. The cross-sections for $\tilde{t}$ and $D$ have to be divided by an additional factor between 1.5 and 4 owing to hadronization effects, as discussed in the text. In (a) and (b) the upper curves correspond to the higher value of $\sqrt{s}$; in (c), the upper (lower) curves are for particles that penetrate (are absorbed in) the calorimeter. Note that in (a) and (b) full azimuthal coverage is assumed, while the special-purpose detector (c) only covers $20^{\circ}$ in both $\theta$ and $\phi$.

Fig. 3: (a) Angular and (b) monentum distribution of signal events for $m=$ $50 \mathrm{GeV}, \sqrt{s}=1.8 \mathrm{TeV}$. Note that $p$ is the particle's momentum before it enters the calorimeter, where it will be slowed down somewhat. The meaning of the different curves is as in Figs. 2. 


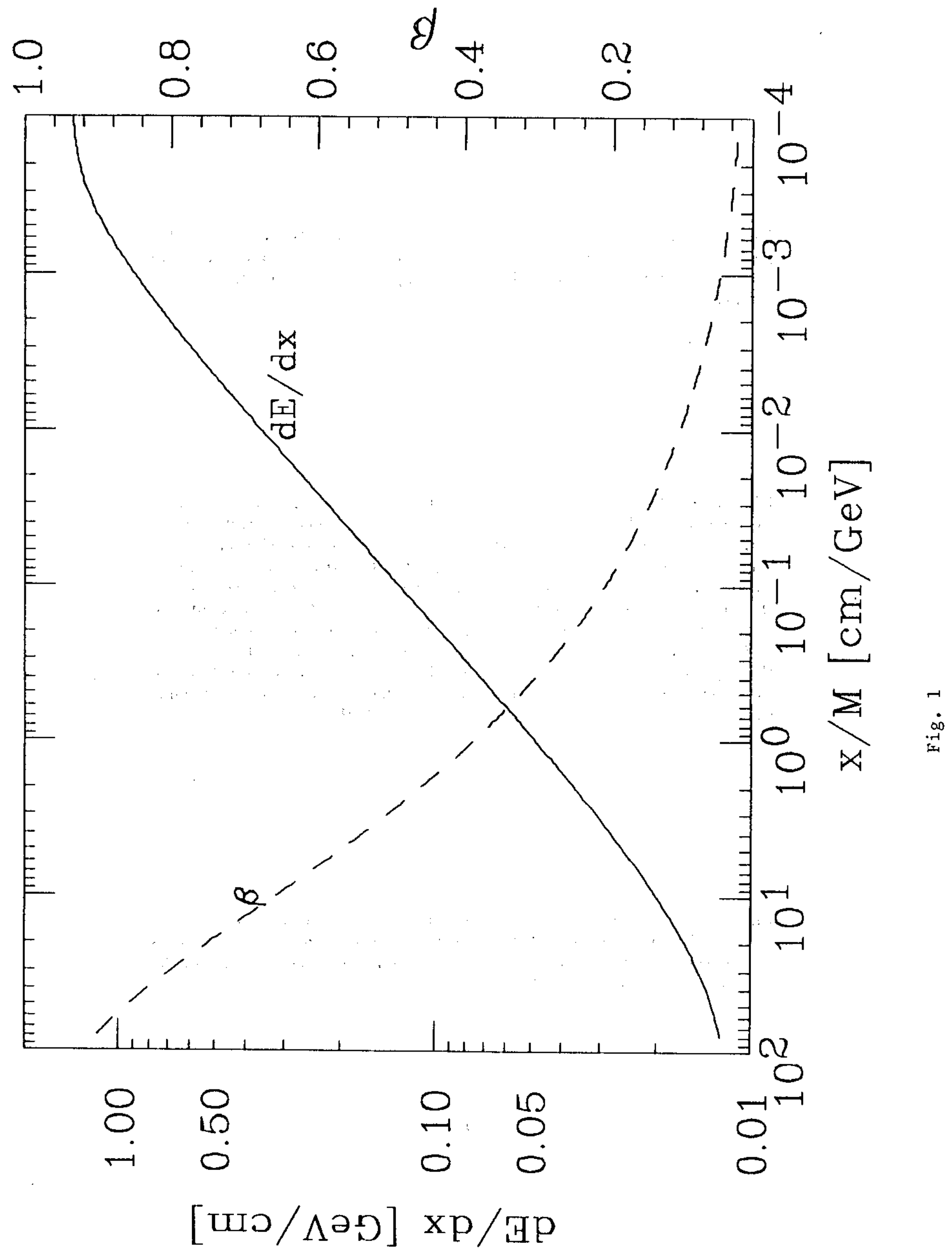




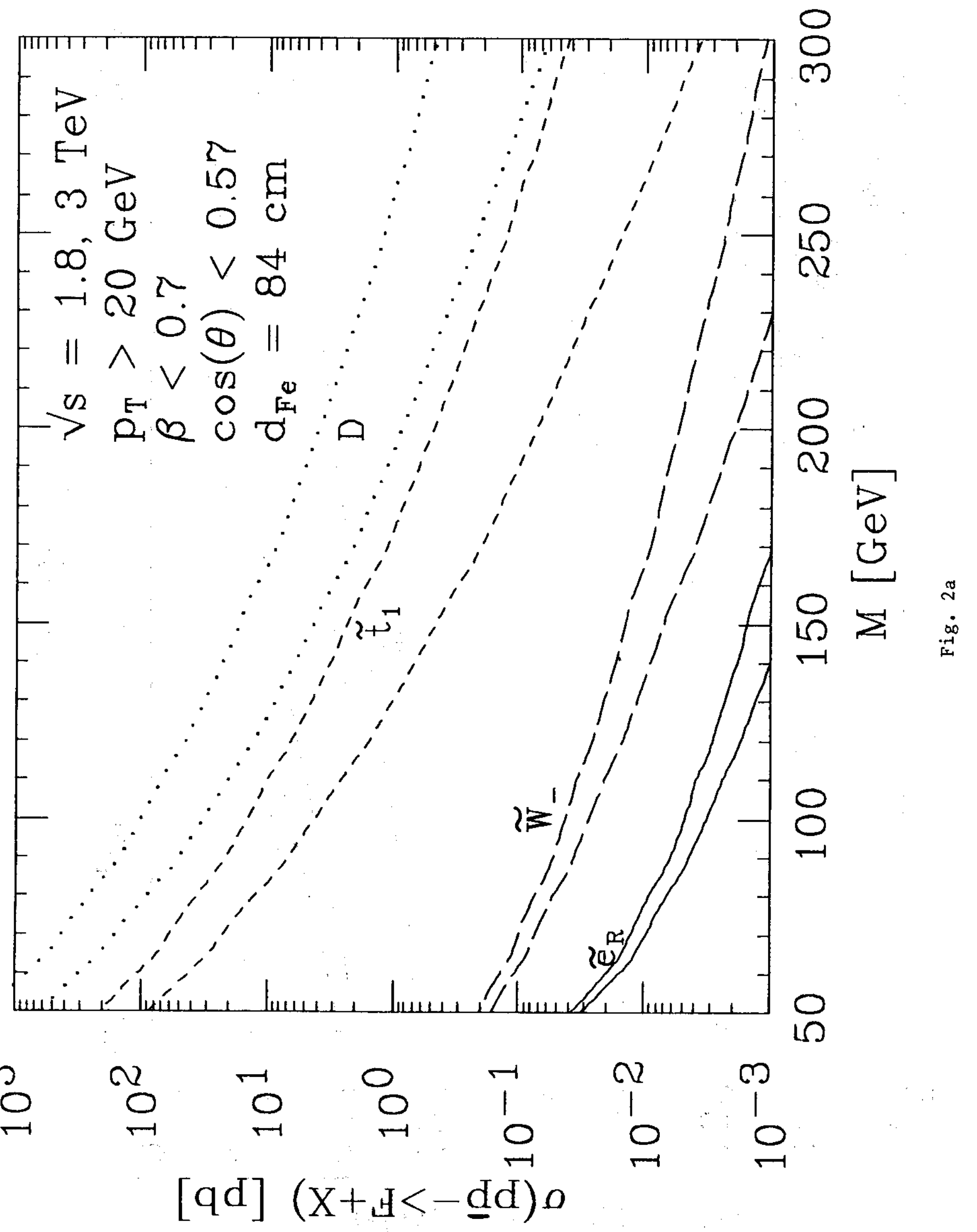




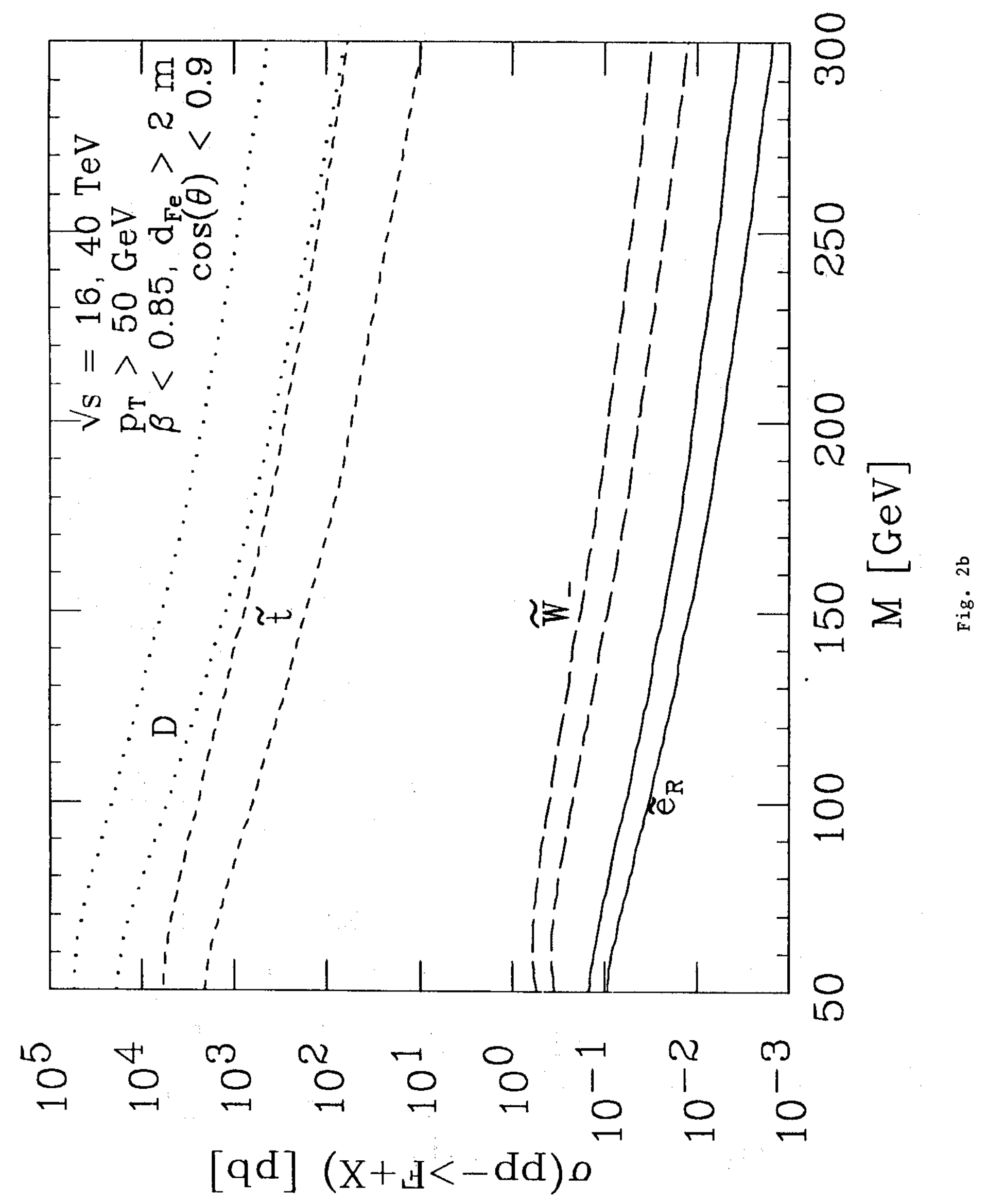



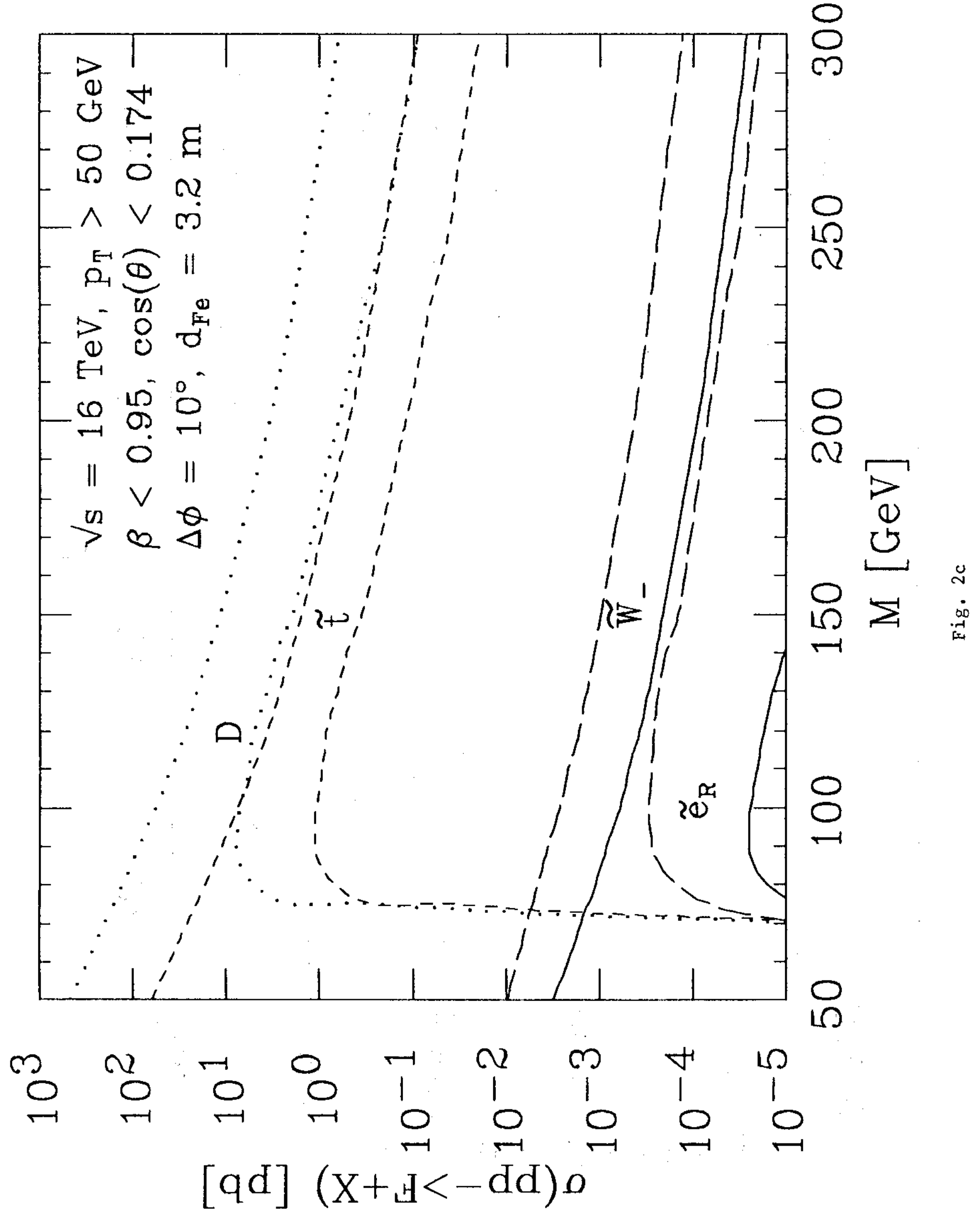


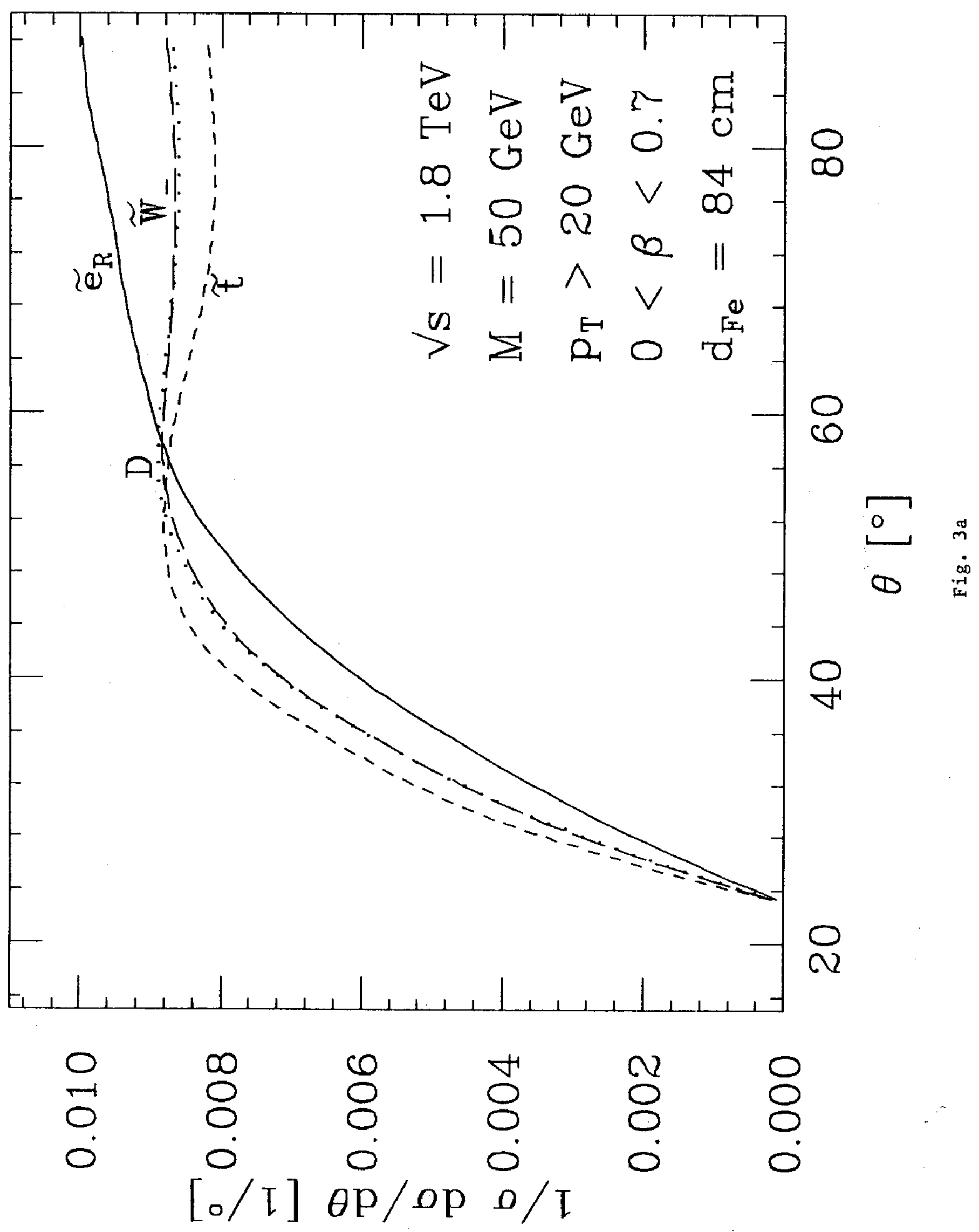




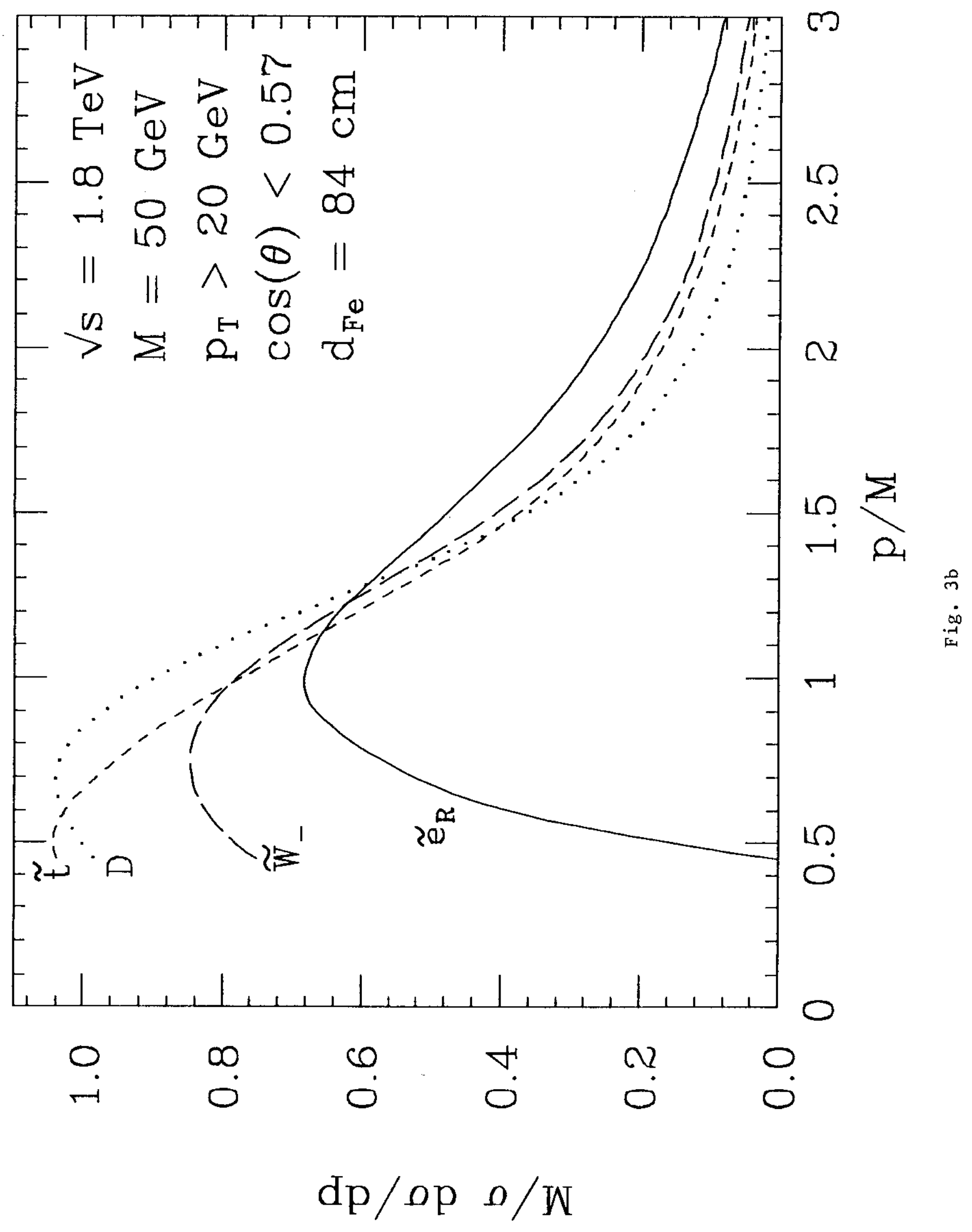

